National Ignition Facility and Managing Location, Component, and State

C. Foxworthy, T. Fung, R. Beeler, J. Li, J. Dugorepec, C. Chang

July 29, 2011

IAEA 8th Technical Meeting San Francisco, CA, United States June 20, 2011 through June 24, 2011 
This document was prepared as an account of work sponsored by an agency of the United States government. Neither the United States government nor Lawrence Livermore National Security, LLC, nor any of their employees makes any warranty, expressed or implied, or assumes any legal liability or responsibility for the accuracy, completeness, or usefulness of any information, apparatus, product, or process disclosed, or represents that its use would not infringe privately owned rights. Reference herein to any specific commercial product, process, or service by trade name, trademark, manufacturer, or otherwise does not necessarily constitute or imply its endorsement, recommendation, or favoring by the United States government or Lawrence Livermore National Security, LLC. The views and opinions of authors expressed herein do not necessarily state or reflect those of the United States government or Lawrence Livermore National Security, LLC, and shall not be used for advertising or product endorsement purposes. 


\title{
NATIONAL IGNITION FACILITY AND MANAGING LOCATION, COMPONENT, AND STATE
}

\author{
Cemil Foxworthy, Tracy Fung, Rich Beeler, Joyce Li, Jasna Dugorepec, Cathy Chang
}

Lawrence Livermore National Laboratory, Livermore, California USA

\begin{abstract}
The National Ignition Facility (NIF) at the Lawrence Livermore National Laboratory is a stadium-sized facility that contains a 192-beam, 1.8-Megajoule, 500-Terawatt, ultraviolet laser system coupled with a 10-meter diameter target chamber. There are over 6,200 Line Replaceable Units (LRUs) comprised of more than 104,000 serialized parts that make up the NIF. Each LRU is a modular unit typically composed of a mechanical housing, laser optics (glass, lenses, or mirrors), and utilities. To date, there are more than 120,000 data sets created to characterize the attributes of these parts. Greater than 51,000 Work Permits have been issued to install, maintain, and troubleshoot the components. One integrated system is used to manage these data, and more. The Location Component and State (LoCoS) system is a web application built using Java Enterprise Edition technologies and is accessed by over 1,200 users. It is either directly or indirectly involved with each aspect of NIF work activity, and interfaces with ten external systems including the Integrated Computer Control System (ICCS) and the Laser Performance Operations Model (LPOM). Besides providing business functionality, LoCoS also acts as the NIF enterprise service bus. In this role, numerous integration approaches had to be adopted including: file exchange, database sharing, queuing, and web services in order to accommodate various business, technical, and security requirements. Architecture and implementation decisions are discussed.
\end{abstract}

Keywords: National Ignition Facility, Location, Component, State

* This work performed under the auspices of the U.S. Department of Energy by Lawrence Livermore National Laboratory under Contract DE-AC52-07NA27344.

\section{Business Functionality}

LoCoS supports the work activity of all parts of the NIF organization including: the Control Room, technicians who install and maintain LRUs and components, "factory" operations that build and service LRUs and components, and management.

\subsection{Seating Chart, Logs, and Work Permits}

LoCoS depicts the NIF via a series of seating charts. At the lowest level, each LRU is represented as a box on a diagram. A top-level view shows the facility broken down into major areas: Laser Bays 1-2, Capacitor Bays 1-4, Switchyards 1-2, and the Target Bay. Selecting one of these allows the user to see a rolled-up view of the area. For example, each of the 96 beams in the Laser Bays is given a color code and relevant icons. The color indicates commissioning status of empty, installed, installation qualified (IQ), or operational qualified (OQ). Icons indicate whether the use of a LRU is restricted, or if there is a LRU installed that is not the primary type e.g. a shield. At the summary level, these colors and icons are aggregated for all the underlying LRUs in the beam. Once a particular beam is selected, the detail view is presented, with a separate box for each LRU. The boxes in the view have colors and icons relevant to the particular LRU. Further detail is available if a particular LRU is selected. This includes the specific list of restrictions applicable, the bill of materials (BOM) including part numbers and serial numbers, and the history of transactions like installation, removal, IQ, and OQ.
There are 6 types of Logs - Restrictions, Problems, NCRs (Non-Conformance Reports), Actions, Operations, and Notes. As mentioned above, restrictions resolve as icons on the seating chart. These logs indicate use restrictions ranging from parameters for limited use to out of service conditions. Problems Logs record issues that occur during shots or other activities. The NIF is broken down into sub-systems to which a Problem Log is attributed. This causes it to be assigned to the appropriate sub-system manager for action and resolution. NCRs are similar. They indicate an out-of-specification condition for a particular serial number. Action logs assign to an individual, a particular action to be taken, possibly in response to a Problem Log. Operations Logs summarize activities related to shot preparation and shot operations. Notes are, typically, comments or status updates to other logs such as Problem or Restriction Logs. Each log can be associated with one or more other logs of any type.

An important LoCoS feature that drives daily work at the NIF is the Work Permit. All work done in the facility, such as maintenance, LRU installation/removal, configuration, and even software deployments, is governed by the Work Permit process. Here the work is described, the individuals performing the work are identified, the dates and times of the work period are specified, the sub-systems affected are listed, and electronic signatures are acquired for approval of the work. The system uses information such as which safety systems are affected to determine what kinds of approvals are required. NIF workers cannot start their 
work until the Work Permit is released. Status notifications can be sent via email or pager.

\subsection{Orders}

While a separate Enterprise Resource Planning (ERP) system is the system of record for parts, serial numbers, BOM, and assembly/installation/removal transactions, LoCoS acts as the front-end to these data and transactions for most NIF users. Work Orders are used by factory technicians to issue component serial numbers to assemblies and LRUs, and complete routing steps. Routing steps represent individual activities needed to complete the assembly work. Some routing steps may require the upload of a data set, which is discussed below. Once the Work Order is completed, the associated LRU can be installed into the facility. An Install Order is used for this, and the transaction is reflected in the seating chart. Depending on the LRU type, an IQ and/or OQ may be required. Commissioning Service Orders are used for this purpose. These involve routing steps, data set uploads, and data transfer to ICCS. To complete the life cycle, Remove Orders are used to take a LRU out of the facility. This could be due to end of life, maintenance, or shot configuration requirements.

In addition to orders, serialized components can be searched by serial number, part number, or part type. Their current status (work in progress, inventory, or installed), location, hazardous material/contamination/radiation levels, logs, orders, and data sets can be viewed.

\subsection{Data Sets}

LoCoS data sets come in two types: Item Inspection Data Sets and Calibration/Metrology Data Sets. Item Inspection Data Sets are further categorized as either simple file attachments, or customized optic inspections, which include metadata and images. In either case these data sets are associated with a serial number. Calibration/Metrology Data Sets involve predefined Microsoft Excel or Hierarchical Data Format [1] files that are parsed when uploaded to store name/value pairs in the database. These can be associated with serial numbers, ICCS control points, or part numbers. Those associated with part numbers are considered specifications, and have minimum and/or maximum values. These are then used to compare to measured values for serial-based data set uploads with the matching part number. Values outside of specifications are marked, and a Non-Conformance Report (NCR) can be attributed to them, if desired. Some data sets are associated with Work Order or Service Order routing steps. The system may require a data set upload before completion of the routing step. This ensures the appropriate data capture for NIF final optics, target diagnostics, and targets. A subsequent routing step may cause the captured data to be transferred to ICCS for use in adjusting final optic position, or aligning a target diagnostic or target when installed. One can search for uploaded data sets by serial number from a data set search screen. Alternatively, the seating chart can be used to drill into a particular LRU in order to select serial numbers off its BOM to get to the relevant data sets.

\subsection{Architecture}

LoCoS is a Java Enterprise Edition web application composed of eight modules and makes use of multiple databases (see figure 1). Each module is implemented as its own application into one of five Oracle OC4J Java EE containers. Within the Java space, these technologies are used: Spring Framework, TopLink, Apache Struts, and Ajax. The various modules communicate with each other via web service calls. Some module to module interactions are handled as simple HTML links. Oracle PL/SQL packages are used for some data-centric logic.

LoCoS owns two database schemas and shares five others. One of these shared databases is an Oracle Content Management (CM) schema. This is used to store file attachments for all sorts of entities including: logs, Work Permits, Data Sets, and Orders.

Besides interactive web pages, there are also backend processes. Some of these run on a scheduled basis, such as email notifications to owners of expired Work Permits. Others are responses, requests, or notifications received from external systems.

Another feature provided to LoCoS users is an ad hoc reporting capability. An Oracle Report Server provides this functionality. Some reports are run from a centralized report screen, while others are integrated into various LoCoS application screens. One report is run as a daily extract, the output of which is used by an external system.

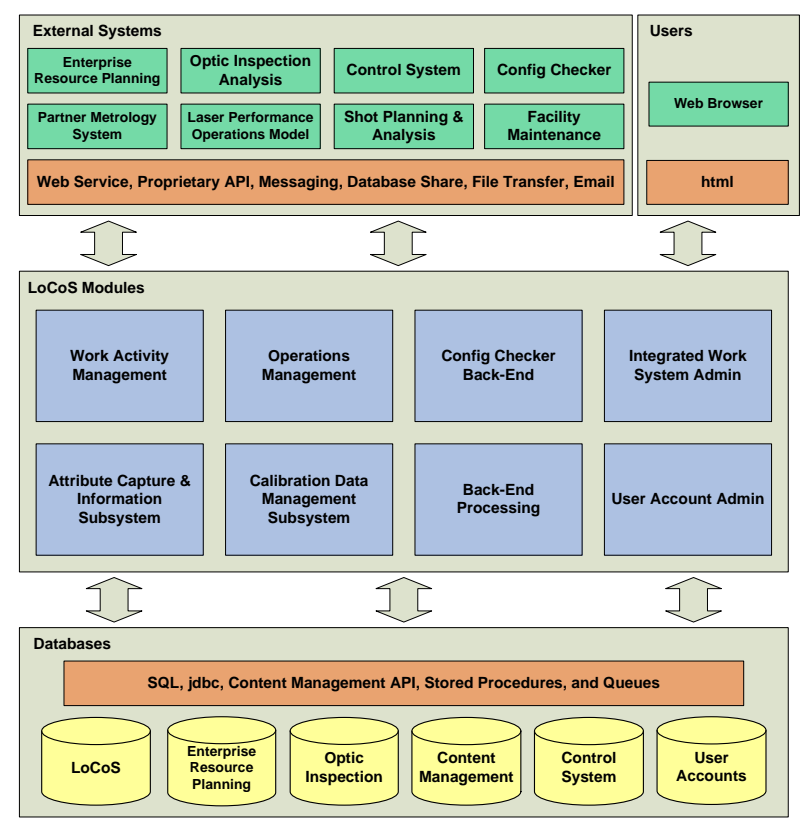

Fig. 1 LoCoS Architecture

\subsection{External Interfaces}


Interfaces to external systems are a key part of LoCoS capability. These have been added over a number of years, and as a result, use different implementations. When the interfaces are depicted as in figure 2, LoCoS takes on the look of a data exchange backbone or Enterprise Service Bus (ESB). While it does not strictly adhere to rigorous ESB principles, LoCoS does provide a similar capability.

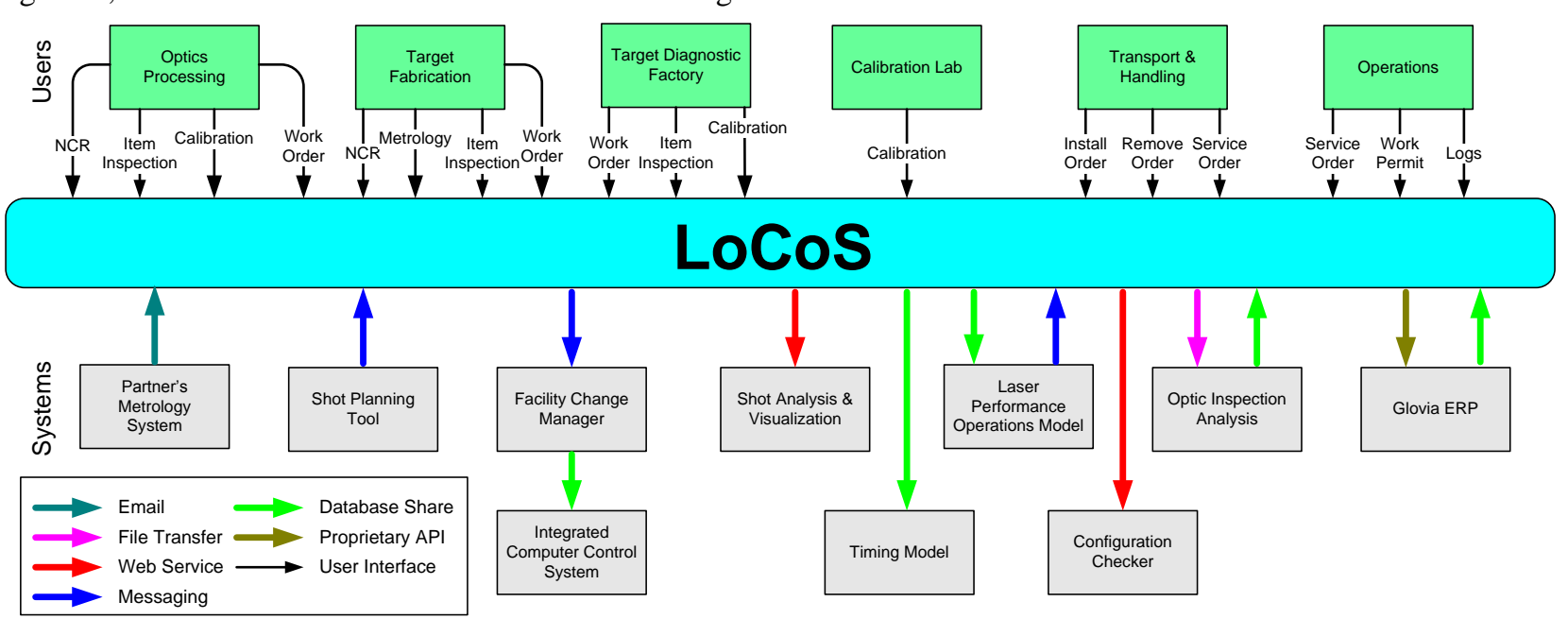

Fig. 2 LoCoS as a Data Exchange Backbone

\subsection{Integration Methods Used}

Some of the simplest interfaces are database shares. Here, the external system's database is queried directly by LoCoS or the external system queries LoCoS' database. This approach is quick to implement, but couples the two systems involved. This is somewhat mitigated through the use of database views, which hide actual database table and column names of the external system from LoCoS.

When data needs to be packaged and processed as a unit of work, File Transfer can be used. Although it gives up some benefits like guaranteed delivery compared to messaging, it is simpler to implement, and can handle large files (e.g. greater than $1 \mathrm{~GB}$ ). Optic Inspection Data Sets are transferred this way (see figure 3 ). The payload typically includes two parts. One is a metadata file that contains some name/value pairs in a text format. The other is an image file in TIF format. A third file rounds out the set. This is a zero byte "flag" file that is written to the file system last by LoCoS. Its presence indicates the payload files are ready to be processed by the external system. Because LoCoS generates a unique reference ID for each transaction, which both systems use, LoCoS can look up the status of file transfer transactions via a database share query.

Email can be a practical interface for certain cases. For LoCoS, this has been the adopted approach to receive files from a NIF partner which supplies subcomponents. These parts have metrology data sets created off-site at the partner's location, which need to be added to LoCoS. Entry via the email server provides the benefit of a virus scan for an added measure of security. The full implementation includes a message queue downstream of the email receipt. LoCoS validates the file contents before accepting the data set. Rejected messages are logged, and can be reprocessed, when corrections are made.

author's email: foxworthy3@llnl.gov
One external system dictates the use of its proprietary Application Programming Interface (API).

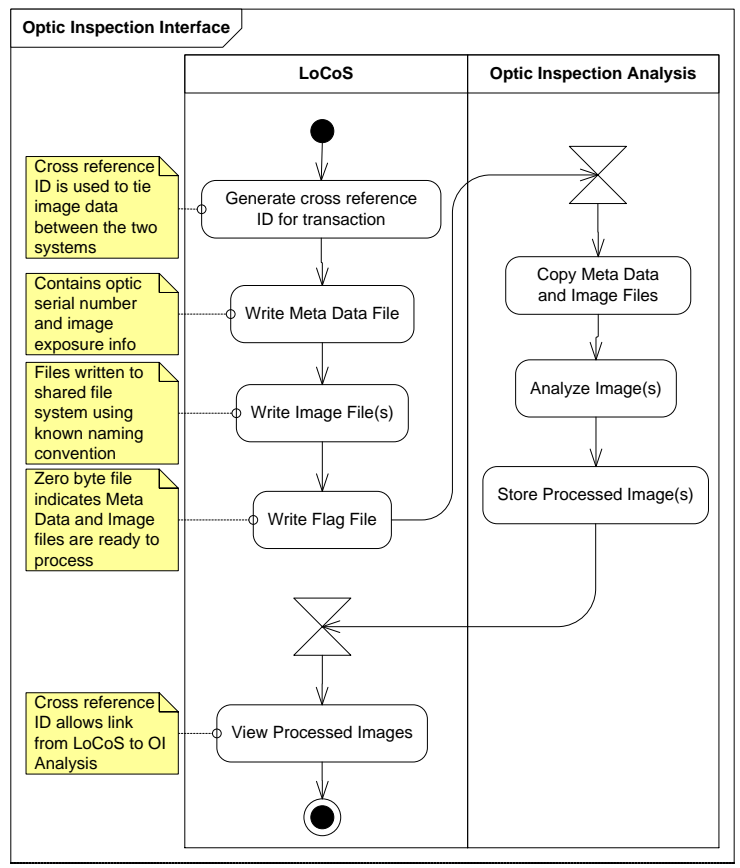

Fig. 3 Optics Inspection Interface utilizes file transfer

There is a Java implementation of the API that is used by LoCoS. This has allowed significant collaboration with the ERP system. Custom transaction functions written for LoCoS on the ERP side can be called by including a library that defines the API.

Web Service implementations have been preferred for synchronous communication to both external systems and between LoCoS modules. Here the system making the request requires the response right away, and will wait for the responding system. An example of this is the Configuration Checker interface (see figure 4). 
The Configuration Checker makes a web service call using a XML document. This document includes a list of LRU locations to check for required sub-components for a particular experiment. LoCoS performs database queries to get the current configuration of those locations. Then, it responds with a XML document of its own containing the results.

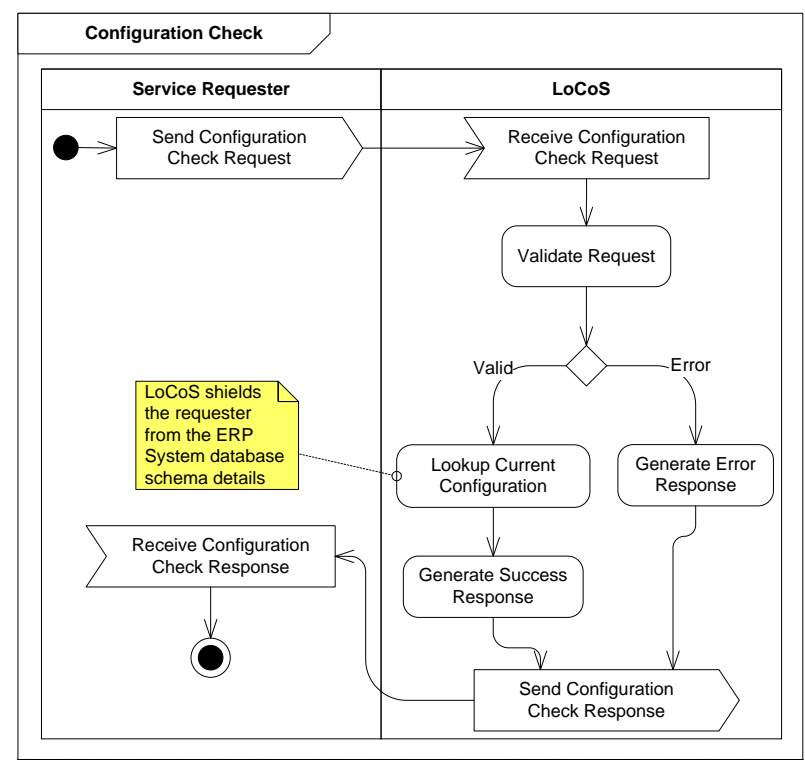

Fig. 4 Configuration Check is done by Web Service

Messaging has been the preferred implementation for asynchronous communication. This approach has been used when a two-way interface is implemented with a request and response, but it is known that the system processing the request will take some time to complete its work [2]. Instead of blocking the requesting system while waiting for the response, the asynchronous nature of the implementation allows the requests to be enqueued, with the expectation that the response can be found some time later in another queue (see figure 5). The request and response messages are associated by the use of a unique request id, which is referred to in the response message. Of course, one-way interfaces are amenable to this method as well. In such cases, there are request messages without corresponding responses.

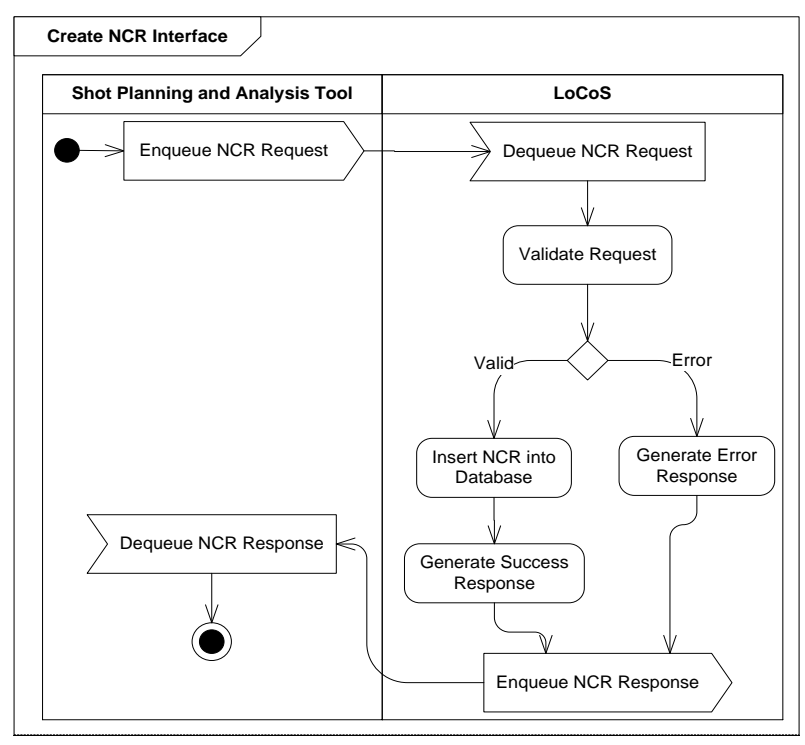

Fig. 5 The Create NCR Interface uses messaging

\section{Future}

Two paths of future development are evident. First, added business functionality will be implemented. With the anticipation of the NIF increasing its role as a user facility [3], new LoCoS features will be needed to support those efforts. The second area will be to increase the technical sophistication of the application. While the existing implementations of the external interfaces are functioning, there are gains to be made. A transition from the hub-and-spoke model to an ESB model would benefit the reliability of the existing interfaces, and provide an extensible framework for the future interface requirements sure to come [4]. Some of the groundwork has been laid for this change through the use of messaging and configuration-driven interface implementations. The possibility of separating the LoCoS business functions from the external interface infrastructure lays ahead.

\section{Acknowledgements}

The authors would like to thank Laura Foxworthy and Allan Casey for their helpful edits. Microsoft Excel is a registered trademark. This work is performed under the auspices of the U.S. Department of Energy by Lawrence Livermore National Laboratory under Contract DE-AC52-07NA27344.

\section{References}

[1] The HDF Group web site, http://www.hdfgroup.org

[2] G. Hohpe, R. Woolf, Enterprise Integration Patterns: Designing, Building, and Deploying Messaging Solutions, Addison Wesley, 2003

[3] E. Moses, C. Keane, R. Al-Ayat, B. Remington, G. Collins, "Inertial Confinement Fusion and High Energy Density Science Experiments at the National Ignition Facility," ???

[4] D. Chappel, Enterprise Service Bus, O’Reilly, 2004 